\title{
SOME SPECIAL CONSIDERATIONS IN NEONATAL
}

\section{ANAESTHESIA}

\author{
A. B. BuLL, F.F.A.R.C.S. \\ Professor of Anaesthetics, University of Cape Town \\ Red Cross War Memorial Children's Hospital, Cape Town.
}

STUDIES of mortality associated with anaesthesia and surgery consistently indicate a considerably higher death-rate in which anaesthesia is, or may be, contributory, during the first decade than in the age-groups 10-60 years. In the geriatric age groups, mortality is again high, approaching or equalling that found in the first decade. Stevenson, Reid and Hinton (1953), in a study of 1,200 cardiac arrests during anaesthesia, reported 21 per cent of these as occurring in the first decade. West (1954), reported seven cardiac arrests in the 1-10 year age group out of a total of 30 . Beecher and Todd (1954) drew attention to the increased vulnerability of infants. Bergner (1955), found six out of 17 cardiac arrests to be in children under 10 years and Schull (1959), gives a figure of 25 per cent for the same age-group. Kok and Kitay (1960), showed a figure of 16 per cent of 300 cardiac arrests occurring in the 1-10 year age group and Harrison (1965) found that whereas children under 10 years represent 10 per cent of the sungical population subjected to anaesthesia, they comprised 21 per cent of the deaths in which anaesthesia was a contributory cause. Accurate evaluation of these figures is difficult, as only too frequently no attempt is made to separate causative factors into those due to patient disease, those due to surgical management and those due to anaesthetic management, and the background surgical population from which they are taken is ill-defined. However, it would appear that ample justification exists for the view that the surgical and anaesthetic care of small children is a matter for special consideration. This view is strengthened by Smith (1954) in a detailed analysis of deaths occurring in the individual years of the first decade. He found a 3.9 per cent operative and post-operative mortality in the 0-1 year group, this figure being about four times that of any other year in the first decade. Similarly, at the Red Cross War Memorial Children's Hospital, Cape Town, during 1963 and 1964, neonates comprised 1.8 per cent of deaths occurring during operation or in the post-operative period, as against 0.9 per cent mortality associated with surgery in all children from 0-11 years of age.
Once again, the causes for this high mortality, compared with other age-groups, cannot be easily or clearly separated into those due to anaesthetic factors and those due to surgical factors. The operative care of infants, to give greatest success, depends largely upon teamwork, mutual appreciation of problems amongst members of the team and a degree of technical skill above that required when dealing with older age-groups. The application of methods and knowledge derived from anaesthesia in adults or older children to neonates without modification based on their special requirements will inevitably lead to complications and increased mortality. Pre-operation preparation, detailed anatomical and physiological differences, modified drug response, specialised pathology of neonatal disorders and the description of specialised techniques to deal with special conditions, and methods of monitoring during anaesthesia will not be discussed.

It is proposed here to deal only with three of the main considerations which require special attention, other than the ordinary principles of anaesthesia. These are: -

1. Pulmonary ventilation.

2. The control of body temperature.

3. Accurate assessment and replacement of blood loss during operation.

\section{Pulmonary Ventilation}

There are important differences in the respiratory physiology of neonates as compared with adults and older children. As one of the anaesthetist's functions is to maintain adequate respiratory exchange, an appreciation is essential. Whereas much has been written about respiratory physiology in the adult, both in the normal and abnormal states, the same does not hold for neonates. This is due largely to the technical difficulties involved in making respiratory measurements on these small patients. With regard to the mechanical aspects of breathing, one finds that the neonatal thorax presents a combination of structural handicaps to ventilatory efficiency. The thoracic cavity is small. The sternum, being pliable, provides an unstable base for the ribs. The ribs themselves, being 
horizontal, do not lend themselves to the "bucket handle" movement which the thoracic respiratory muscles provide in the older child and adult. The intercostal muscles are poorly developed and the accessory musoles of little assistance. Ventilatory volume change is brought about largely by the diaphragm, and because of this, severe embarrassment to respiration can and does occur with abdominal distension from any cause. This is important to bear in mind also in such operations as repair of omphalocele and large umbilical herniae, where return of viscera and too "tight" a repair may result in respiratory distress. This will not be evident during the surgical procedure if controlled ventilation is being used, and may only manifest itself in the post-operative period.

The compliance of the thoracic cage is far greater in infants, and this gives rise to the characteristic sternal and thoracic recession seen with even mild degrees of respiratory obstruction during spontaneous respiration. This is frequently seen even when no organic obstruction is present, but when there is increased respiratory demand, e.g., during crying, or during overbreathing in states of metabolic acidosis. It may be taken as visible evidence of the diminished respiratory reserve of small infants, the picture of this recession of the rib cage being mechanically and physiologically similar in effect to flail chest injury in adults. McDonald (1960) has stated that in comparison with the adult, the newborn has half the respiratory exchange surface per unit lung weight and one third the respiratory exchange surface per unit body weight as compared with the adult. This is combined with a comparatively high metabolic rate, making a rapid respiratory frequency necessary to compensate for these deficiencies. High respiratory rate implies the frequent shifting of dead space air as a concomitant. Nelson, Prod-Lom, Cherry, Lipsitz and Smith (1962), calculating dead space from $\mathrm{CO}_{2}$ tensions in expired and alveolar air arrived at a figure of $4.4 \mathrm{ml}$. this being some 30 per cent of tidal volume. This percentage is not far different from that found in adults, but it is of importance to emphasise the fact that in terms of absolute values, $5 \mathrm{ml}$. dead space in a neonate is comparable with about $100 \mathrm{ml}$. in adults. Even a small increase in anatomical dead space volume such as that which can be produced by poor choice of anaesthetic circuit or apparatus, becomes very significant under these circumstances and can decrease the already handicapped respiratory reserves of these small patients. It may be argued that slight increase in dead space can be compensated for by increase in ventilation, but this can only be so, under most conditions in anaesthetic practise, if fresh gas flow is increased proportionately to match the increased tidal volume. Even this increase in ventilation may not fully compensate for the added dead space. Clappison and Hamilton (1956), showed that increasing the dead space in normal unmedicated subjects led to increase in tidal volume and rate which were not sufficient to correct end-expiratory $\mathrm{CO}_{2}$ tension. The problem of apparatus dead space in paediatric anaesthetic apparatus has been well summarised by Voss (1963) in an assessment of the Magill, Potter and Cape Town gas circuits applied to paediatric practice. The Cape Town system has been found to be the most suitable for small children and also has the virtue of extreme simplicity and versatility. This system, (Voss, 1963), was evolved in 1954 at the Red Cross War Memorial Children's Hospital and is used here as standard apparatus for neonates and small children. It consists of a standard angle piece with a tube of $6 \mathrm{~mm}$. internal diameter soldered in the position shown in Fig. 1. A $75 \mathrm{ml}$. soft latex bag with an open tail is fitted over the open limb of the angle piece. A similas arrangement can be used for endotrache ? anaesthesia and has been described by Res. (1950). These systems are, in effect, "T-piece" circuits and require fresh gas-flows of at least double the minute volume of the patient. Being without valves they offer very little resistance $\stackrel{\mathbb{Q}}{\circ}$ to respiratory flows.

The widespread use of muscle relaxants and $\stackrel{9}{\Im}$ controlled respiration necessitates some knowledge of the ventilatory requirements in terms of volume and pressures which may be tolerated by the infant and of the qualitative and quan- 3 . titative requirements in terms of gas exchange. The accurate assessment of adequate respiratory exchange is not easy unless facilities for frequent $ᄋ$ blood-gas analysis or end expiratory $\mathrm{O}_{2}$ and $\mathrm{CO}_{2}$ measurements are available. In general, 9 it may be stated, however, that if a patient with

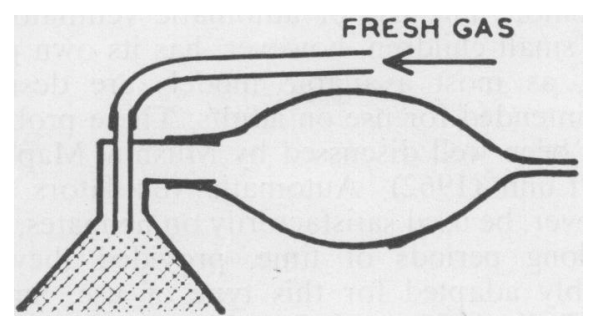

Fig. 1.-The Cape Town System. 
a normal haemoglobin value and normal circulatory and respiratory system, breathing air, shows no sign of cyanosis, the blood carbon dioxide tension is probably not increased. Under conditions of anaesthesia, however, decreased ventilation may occur in the presence of high inspired oxygen tensions leading to the insidious development of respiratory acidosis, which, if superimposed upon possible pre-existing metabolic acidosis may lead to fatal decrease in arterial blood $\mathrm{pH}$ and become a factor in mortality. Conversely, overventilation over a long period of time can lead to marked lowering of $\mathrm{PcO}_{2}$ and rise in $\mathrm{pH}$ accompanied by a rise in cell potassium level and fall in extracellular potassium. If facilities for gas analysis are not available, a reasonable estimate of ventilatory requirements may be made from the patient's size. Radford (1955), have produced a most useful nomogram for this purpose from which it is possible to estimate required respiratory frequency and tidal volume from body weight. From this it will be seen that tidal volume of about $15-20 \mathrm{ml}$. at a frequency of $30-40$ per minute is about normal for the neonate. The construction of the nomogram is based on three major assumptions. Firstly, it assumes that basal carbon dioxide production can be predicted from body weight. Secondly, that respiratory dead space can be predicted from body weight and is, in fact, about $1 \mathrm{ml}$. per pound. Thirdly, that optimal arterial $\mathrm{PCO}_{2}$ is $40 \mathrm{~mm}$. $\mathrm{Hg}$. These assumptions must be borne in mind when referring to it. Measurement of these small tidal volumes is, however, extremely difficult, as most conventional dry gas meters suitable for operating theatre use show undesirable inaccuracies at the small flow-rates encountered with these small volumes. No simple satisfactory method has yet been devised to overcome these difficulties, and in practice, the use of a monaural stethoscope strapped to the patient's chest will at least give audible indication of air entry. The nearest approach to measured ventilation in neonates is probably provided by the use of a volume-cycled ventilator. The use of automatic ventilators in very small children, however, has its own problems, as most available models are designed and intended for use on adults. These problems have been well discussed by Mushin, Mapleson and Lunn (1962). Automatic ventilators may, however, be used satisfactorily on neonates, even for long periods of time, provided they are suitably adapted for this type of use. Smythe and Bull (1959) and Smythe (1963), describe the use of the Radcliffe respirator in the treat- ment of tetanus neonatorum with IPPR and curarization. For their satisfactory use, the essential thing to remember is that the unmodified adult ventilator delivers a flow-rate during inspiration which is too high. This has the effect of ensuring turbulent flow in the airway system, thus relatively increasing resistance and interfering considerably with the triggering mechanism of the ventilator. It also carries the danger of overinflation, though this is not great. Rosen and Laurence (1965) give the pressure at which rupture of the neonate's lung occurs as being greater than $50 \mathrm{~cm} . \mathrm{H}_{2} \mathrm{O}$. Most automatic ventilators are provided with safety devices which prevent this pressure being reached.

The size and nature of the infant's respiratory tract makes it particularly vulnerable to mechanical obstruction. The large antero-posterior diameter of the head in relation to that of the thorax makes kinking of the soft trachea and obstruction in the pharynx occur very readily unless hyper-extension of the neck is maintained. The small diameter of the larynx and trachea, smallest at the level of the cricoid cartilage, allows little latitude for dimunition of the lumen by secretions, oedema or endotracheal tubes. Not often realised to the full is the way in which badly chosen endotracheal tubes can increase airway resistance and cause respiratory obstruction instead of preventing it. This is due to the fact that under conditions of flow operating in the respiratory tract, the cross section area of the tube or airway is the most important measurement determining flow resistance. Decrease in the diameter of the neonatal trachea with a $4 \mathrm{~mm}$. diameter, such as would arise from the passage of an endotracheal tube with wall thickness of $1 \mathrm{~mm}$. will produce a 75 per cent decrease in cross section area. This is illustrated in Fig. 2 and applies equally to obstruction arising from oedema due to trauma or inflammatory processes.

Endotracheal tubes should, therefore, be chosen with the largest possible internal diameter. This, however, presents the problem of kinking of the tube if the wall is excessively thin. To provide both adequate lumen and freedom from kinking, the Oxford pattern (Alsop, 1955) neonatal endotracheal tube is most satisfactory. This tube has a tapered wall, but a constant lumen, providing rigidity of the section where kinking is most likely, whereas the end section which lies in the larynx and trachea has an extremely thin wall. In addition, the tube is moulaed with a right angled curve to fit the curve of the oropharynx. Other tubes having a thin-walled section which fits the 
OBSTRUCTION CAUSED BY DIMINUTION OF CROSS SECTION AREA.

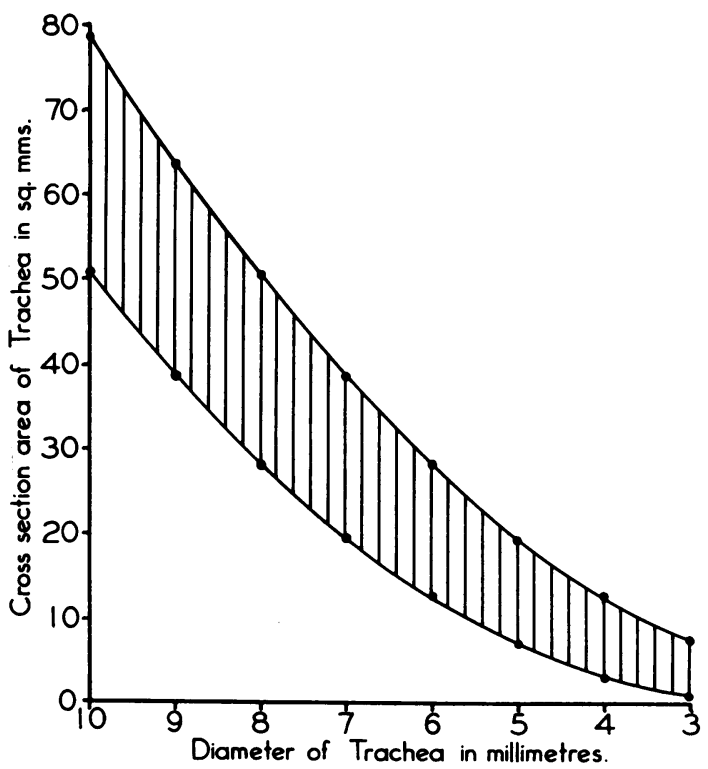

FIG. 2.-The upper curve is a plot of $\pi \mathrm{r}^{2}$ against tracheal diameter. The lower curve represents $(\pi \mathrm{r}-1)^{2}$ against tracheal diameter. The vertical distance between the curves at any diameter represents obstruction. The vertical distance below the lower curve at any value of diameter represents free lumen.

larynx and trachea but with reinforced walls proximally are obtainable in rubber or in plastic and have been described by Cole (1954) and Tunstall (1961). The funnel shape of the infant larynx, with the narrowest position at the cricoid ring allows an airtight fit between endotracheal tube and trachea, if the correct size of tube is chosen, making the use of cuffed endotracheal tubes unnecessary. Indeed, it is impractical to attempt the use of cuffed tubes in small babies as the construction of such a tube leaves a size of lumen which will lead to almost total respiratory obstruction. Specifications for endotracheal tube sizes have been drawn up and published in the British Standards Institution Specification B.S. 3487 of 1962.

As important as the choice of a tube with an adequate lumen is the choice of an endotracheal tube adaptor which does not cause a constriction in the anaesthetic system. The lumen of the adaptor must be at least as great as that of the tube.

\section{The Control of Body Temperature}

Accidental hypothermia occurring in the new- $\overline{3}$ born is a well-known occurrence. This has been $\stackrel{\AA}{\AA}$ attributed to immaturity of the heat-regulating.. mechanism making the neonate behave like a $\vec{F}$ poikilotherm, rather than a homeotherm, until thermoregulatory control develops with increas- $\frac{C}{0}$ ing maturity. It is likely, however, that the $\overline{\frac{\sigma}{5}}$ thermal instability of the newborn compared $\widetilde{\nabla}$ to the adult is due mainly to physical characteristics affecting heat loss. The body-weight ${ }^{\infty}$ of the newborn infant is about 5 per cent of $\vec{O}$ that of the adult, while its surface area, by $\doteq$ comparison, amounts to approximately 15 per cent. This high surface-area to body-mass ratio, and its curved body surfaces greatly facilitates 3 heat exchange between the infant and its environment by conduction, convection and radia- $N$ tion. Insulation of body core provided by skin $\stackrel{\infty}{\circ}$ and subcutaneous fat is significantly less in the $\omega$ newborn, too, again increasing heat exchange 0 by conduction. A further important factor in 은 the control of heat dissipation depends on the circulation. Blood flow to the periphery plays $\subseteq$ a large part in the transfer of heat from the $\overparen{\Phi}$ body core to the surface, when heat loss or $\vec{\theta}$ gain takes place. This blood flow is, in ture regulated by vasoconstriction and vasodilat tion, both functions being well developed in th neonate. Heat production depends on the ability to increase metabolic rate in response to cold, and the unanaesthetised neonate does, in fact, $\stackrel{\mathbb{D}}{\mathbb{D}}$ respond in this way. (Bruk, 1961; Adams, Fujiwara, Spears and Hodgman, 1964.)

During anaesthesia, a number of factors may combine to render thermoregulation inefficient. Tissue metabolism depends upon adequate supplies of oxygen, glucose and other suitable substrates. Cross, Tizzard and Trythall (1958) have shown the effects of anoxia in this respect. 3 Infants rendered hypoglycaemic through im- o proper pre-operative preparation show a very marked fall in body temperature during anaesthesia. Drugs used in anaesthesia certainly play a part. The state of anaesthesia itself abolishes muscular activity to a large extent, or even N entirely, so greatly diminishing a big source of ${ }^{\circ}$ heat production. Many anaesthetic agents, not- $\tilde{0}$ ably halothane, ether and cyclopropane, produce $\underset{\omega}{\omega}$ vasodilatation of the infant's surface area, large in relation to its body mass. Hypnotics, anal- 6 gesics, neuromuscular and autonomic nervous blocking agents almost certainly all contribute $\stackrel{\mathscr{\rho}}{+}$ towards impairment of thermal perception and 7 the central control of heat production. Placed in conditions of low ambient temperature with 
which its deranged thermoregulatory mechanisms and physical handicaps cannot cope adequately, the body temperature of the anaesthetised infant will invariably fall unless adequate steps are taken to prevent this. The transfusion of cold blood can also play an important part, as during major surgery a considerable percentage of the patient's blood volume may need replacing. Major exposure of thoracic or abdominal viscera will also play a part in increasing the surface from which heat may be lost.

The degree to which body temperature may fall, together with a statistical analysis of the main factors contributing to this fall has been reported by Harrison, Bull and Schmidt (1960). Farman (1962) stresses the importance of inadvertent hypothermia in anaesthetised infants as a contributing cause to mortality. On the other hand, the merits of cooling seriously ill infants under certain circumstances, have been put forward by MoCredie (1962). As far as anaesthetic management is concerned, however, most experienced paediatric anaesthetists agree that fall of body temperature is undesirable. The main reason for this is that much of the danger of cooling is believed to be due to alteration of the action of muscle relaxants, now an almost universal practice. Our experience has shown that even without the use of musole relaxants, cooling of neonates to temperatures below $90^{\circ} \mathrm{F}$ not infrequently results in severe respiratory embarrassment in the post-operative period. A likely explanation of this is that the hypothermic infant recovering from anaesthesia and bringing its thermoregulatory mechanisms fully into operation, may greatly increase its oxygen consumption and respiratory demands to an extent which it is unable to meet adequately, thus leading to a state of respiratory insufficiency.

From the point of view of practical management, it should be assumed that the anaesthetised neonate behaves very much like a poikilotherm. Ambient temperature and humidity will have considerable influence on its body temperature. Hyperpyrexia in infants and children during surgery has been described frequently, most notably by Bigler and McQuiston (1951). In most instances, such descriptions concern conditions of high ambient temperature in operating theatres without air conditioning. The use of atropine is a further factor under these conditions as it prevents heat loss by sweating. Under modern operating theatre conditions, however, a fall in body temperature is the rule and this requires active steps to prevent the attainment of dangerously low temperatures. In all cases of major or prolonged surgery on neonates, some form of temperature monitoring is most desirable. The most convenient is one of the many available electric thermometers available with either rectal or oesophageal thermocouple or thermistor leads. If ambient temperature is considerably below normal body temperature, as much insulation of the patient as is commensurate with satisfactory surgical access should be provided. This may be done by the use of sterile gamgee tissue, and heat dissipation is further aided if plastic sheeting is incorporated in the draping, as this tends to lessen heat loss due to evaporation of moisture. Active warming may have to be resorted to and this is best achieved by placing the patient on a blanket in which plastic or rubber tubes are incorporated through which warm water may be circulated. Several such blankets are available commercially for this purpose. It is essential, however, that the temperature of the circulating water be controlled by a reliable and accurate thermostat, or burning of the patient may result. Our experience is that a circulating water temperature of $102^{\circ} \mathrm{F}$ should not be exceeded. A warm base such as this provides a warm atmosphere beneath the drapes and is most effective in maintaining body temperature at near normal levels. Prior to the availability of such sophisticated circulatirg water blankets, it was our practice to place the patient on hot water bottles at $102^{\circ} \mathrm{F}$. This is very effective, too, as these provide a heat reservoir, effectively retarding temperature-drop for most procedures of average duration. Cooling due to the administration of cold blood is an important factor to be guarded against. Donor blood is stored at temperatures between $4^{\circ}$ and $10^{\circ} \mathrm{C}$. Some method of warming it before it enters the patient is most desirable and this will be discussed more fully.

\section{Replacement of Blood Loss}

Experience has shown that clinical assessment of blood loss during surgery is a poor guide to the quantity requiring replacement, almost always leading to undertransfusion. In neonatal surgery, where the margin for error in all respects is small, accuracy of estimation of blood loss and its replacement is of greatest importance. In the fit adult, a blood loss of $500 \mathrm{ml}$. is readily compensated by normal physiological processes. This figure represents about 10 per cent of expected normal blood volume. It has been estimated that the average normal blood volume in infants is in the order of $40 \mathrm{ml}$. per 
pound body weight. On this basis, it will be seen that in an infant weighing $7 \mathrm{lb}$. blood loss of $30 \mathrm{ml}$. will be equivalent to $500 \mathrm{ml}$. loss in the adult. To those unaccustomed to working with infants, this is often difficult to appreciate, both as regards estimation of quantity lost during operation and with regard to realising the degree of exsanguination which can take place in the pre-operative period if indiscriminate sampling for biochemical purposes is allowed to take place. Our experience is that a loss of 10 per cent of blood volume in the otherwise fit infant is of relatively small significance. Any quantity above this, however, must be replaced at the time it is lost. It is our practice to translate all blood loss into terms of percentage of expected normal volume, taking this at $40 \mathrm{ml}$. per lb. body weight.

Almost any operation on the neonate may cause blood loss sufficient to require replacement. Pretorius (1960) gives an analysis of blood coss in various types of operation on neonates and small children, and Davenport and Barr (1963) divide such blood loss into two groups. Our experience and that of others makes us firmly convinced that no infant should be subjected to surgery unless cross-matched blood is immediately available.

Some means of measuring blood loss is essential as speculation by surgeon or anaesthetist is invariably grossly inaccurate. The means of measuring the loss should be rapid, simple and reasonably accurate and the limitations of the method chosen appreciated. Rickham (1954) has discussed the difficulties arising in such measurements. Of the methods available for estimating blood loss, the colorimetric method described by Alsop, Emery and Zachery (1963) and the gravimetric method described by Pretorius (1960) are the simplest and most practical. The gravimetric method has the disadvantage of requiring the use of dry swabs and of being subject to error due to evaporation on the one hand and contamination with other fluids on the other. Nevertheless, our experience is that provided accurate standardisation of swabs is ensured and due care is taken in weighing, the gravimetric method compares to within a 5 per cent limit with the colorimetric method which is considerably more elaborate.

When an amount of blood requiring replacement is lost, it should be replaced immediately, to prevent the changes in the fluid compartments of the body which follow haemorrhage. On occasion, blood loss may be rapid, requiring rapid replacement. Such rapid replacement cannot be readily achieved via the small cannulae or needles required for the small veins one is dealing with unless provision is made for some form of pressure transfusion. A convenient method of overcoming this difficulty is to administer blood from a syringe connected to the transfusion system by a three-way tap. This also makes for accuracy of administration of the small absolute quantities involved. The dangers of over-transfusion must not be ignored and once more a figure of 10 per cent in excess of estimated normal blood volume should be taken as the limit.

The transfusion of a high percentage of normal blood volume is not infrequently required and this brings with it the dangers of massive transfusion. Citrate intoxication, hyperkalaemia and disturbances of acid base balance due to the unphysiological composition of stored blood should be watched for. Besseling, Bull, du Plessis and Mason (1965) have summarised this problem and drawn attention to the important role played by hypothermia in the genesis of massive transfusion collapse. The rapid replacement of a large proportion of the blood volume by cold stored blood is a potent cause of hypothermia and in addition, may cause selective cardiac hypothermia during rapid transfusions where the transfused blood forms a high propor tion of venous return. Hypothermia may alsao interfere with citrate metabolism, renders the heart less able to withstand alterations in $\mathrm{Ca} / \mathrm{K}$ ionic ratio and may interfere undesirably with acid base balance. Steps should, therefore, be taken to warm blood to near body temperature under conditions of rapid or massive transfusion. The warming of blood must, however, be undertaken with the greatest caution to avoid damage to the red blood cells which will undergo rapid haemolysis at temperatures in excess of $40^{\circ} \mathrm{C}$. Warming may be carried out either by employing an extension of the intravenous tubing immersed in a reliably thermostatted waterbath or by warming the whole blood container prior to use. The former method is most suitable in paediatric transfusion as the absolute flow-rates through the transfusion system are relatively slow and allow good heat exchange from the waterbath if approximately six feet of plastic tubing is used. It must again be emphasised, however, that under no circumstances must any portion of the blood be allowed to exceed $40^{\circ} \mathrm{C}$ at any time. Warming of the whole container should only be done if all the blood is to be used within a period of an hour or two. Warm blood should be administered at once since incubation of stored blood at near body temperature is deleterious 
to its post-transfusion survival. If warming the whole container is the method chosen, again the strictest control of temperatures is mandatory to avoid haemolysis. Waterbath warming is slow and inefficient as the water temperature must not exceed $40^{\circ} \mathrm{C}$. The method of heating by radio frequency induction, described by Besseling and others (1965) is most suitable and has now been perfected to incorporate automatic temperature control.

Finally, it must be emphasised that the route by which blood is to be given must be reliable. It may be impossible or extremely difficult to replace a dislodged needle or intravenous cannula during operation, so a firmly fixed and reliable scalp vein or cutdown drip is essential before surgery is allowed to commence.

\section{Summary}

The necessity for specialised anaesthetic care of the newborn infant is outlined. The reasons for special attention to pulmonary ventilation, control of body temperature and the adequate assessment of blood loss and its replacement are discussed.

\section{REFERENCES}

Adams, F. H., Fujiwara, T., Spears, R., and Hodgman, J. (1964): Temperature Regulation in Premature Infants, Pediatrics, 33, 487.

Alsop, A. F. (1955): Non-kinking Endotracheal Tubes, Anaesthesia, 10, 401.

Alsop, E., EMERY, J. L., and ZAChery, R. B. (1963): Measurement of Blood Loss during Operation, Brit. med. J., i, 125.

BEECHER, H., and ToDD, D. (1954): A Study of Deaths associated with Anaesthesia and Surgery, Ann. Surg., 140, 2.

Bergner, R. (1955): Cardiac Arrest. Some Aetiological Considerations, Anaesthesiology, 16, 177.

Besseling, J. L., Bull, A. B., Du Plessis, J. M. E., and Mason, I. M. (1965): The Rapid Warming of Blood for Massive Transfusion by R.F. Induction, S. Afr. med. J., 39, 137.

Bigler, J. A., and MCQQuiston, W. O. (1951): Body Temperatures during Anaesthesia in Infants and Children, J. Amer. med. Ass., 146, 551.

BRüK, K. (1961): Temperature Regulation in the Newborn Infant, Biol. Neonat., 3, 65.

Clappison, G. B., and Hamilton, W. K. (1956): Respiratory Adjustments to Increases in External Deadspace, Anaesthesiology, 17, 643.

Cole, F. (1945): A New Endotracheal Tube for Infants, Anaesthesiology, 6, 87.
Cross, K. W., Tizzard, J. P. M., and Trythall, D. A. H. (1958): The Gaseous Metabolism of the Newborn Infant Breathing $15 \%$ Oxygen, Acta paediat. (Uppsala), 47, 217.

DAVENPORT, H. T., and BARR, M. N. (1963): Blood Loss During Paediatric Operations, Canad. med. Ass. J., 89, 1309.

FARMAN, J. V. (1962): Heat Loss in Infants Undergoing Surgery in Air Conditioned Theatres, Brit. J. Anaesth., 34, 543.

Harrison, G. G., Bull, A. B., and Schmidt, H. J. (1960): Temperature Changes in Infants and Children During General Anaesthesia, Brit. J. Anaesth., 32, 60.

Harrison, G. G. (1965): Personal Communication.

KoK, O. V. S., and KITAY, C. (1960): Cardiac Arrest Associated with Surgical Procedures, S. Afr. Med. J., 34, 229.

Mc. Credie, D. A. (1962): The Use of Hypothermia in Paediatric Emergencies, J. Paediat., 61, 653.

McDonald, I. H. (1960): Infant Physiology and Anaesthesia, Brit. J. Anaesth., 32, 22.

Mushin, W. W., Mapleson, W. W., and LunN, J. N. (1962): Problems of Automatic Ventilation in Infants and Children, Brit. J. Anaesth., 34, 514.

Nelson, N. M., Prod'hom, L. S., Cherry, R. B., LiPSiTZ, P. J., and Smith, C. A. (1962): Pulmonary Function in the Newborn Infant, Paediatrics, 30, 963.

Pretorius, J. A. (1960): Blood Loss in Paediatric Surgery, Anaesthesia, 15, 4, 424.

RADFORD, E. P. (1955): Ventilation Standards for Use in Artificial Respiration, J. appl. Physiol., 7, 451.

RICKHAM, P. P. (1954): An Investigation of Blood Loss During Operation on the Newborn Infant, Arch. dis. Child., 29, 304.

Rosen, M., and LAURENCE, K. M. (1965): Expansion Pressures and Rupture Pressures in the Newborn Lung, Lancet, ii, 721.

Schull, L. G. (1959): A Review of Cardiac Arrest at Vanderbilt Hospital, Sth. med. J. (Bgham., Ala.), 52, 143 .

SMITH-RoberT, M. (1959): Anaesthesia for Infants and Children. St. Louis: C. V. Mosby.

Smythe, P. A., and Bull, A. B. (1959): Treatment of Tetanus Neonatorum with I.P.P.R., Brit. med. J., ii, 107 .

Smythe, P. A. (1963): Studies on Neonatal Tetanus and on Pulmonary Compliance of the Totally Relaxed Infant, Brit. med J., i, 565.

Stevenson, H. E., Reid, C., and Hinton, J. W. (1953): Some Common Denominations in 1,200 Cases of Cardiac Arrest, Ann. Surg., 37, 731.

Tunstall, M. E. (1961): A Sterile Disposable Neonatal Tracheal Tube, Lancet, i, 146.

Voss, T. J. V. (1963): Deadspace in Paediatric Anaesthetic Apparatus, Brit. J. Anaesth., 35, 454

WEST, J. P. (1954): Cardiac Arrest during Anaesthesia and Surgery, Ann. Surg., 140, 623. 